\title{
Sarcomatoid Carcinoma of the Urinary Bladder
}

\author{
Kenji Shimodaira ${ }^{*}$, Isao Kuroda ${ }^{1}$, Naohiro Kamoda1, Takuya Ishida ${ }^{2}$, Teiichiro Aoyagi1, \\ Masaaki Tachibana ${ }^{2}$ \\ ${ }^{1}$ Department of Urology, Ibaraki Medical Center, Tokyo Medical University, Ibaraki, Japan \\ ${ }^{2}$ Department of Urology, Tokyo Medical University Hospital, Tokyo, Japan \\ Email: ${ }^{*}$ kenji-shimo@tune.ocn.ne.jp
}

Received 3 March 2014; revised 28 March 2014; accepted 2 April 2014

Copyright (C) 2014 by authors and Scientific Research Publishing Inc.

This work is licensed under the Creative Commons Attribution International License (CC BY). http://creativecommons.org/licenses/by/4.0/

c. (i) Open Access

\section{Abstract}

The patient was a 62-year-old female with bladder carcinoma. TUR-BT was performed in March 2010, and the pathological finding was UC, G2 > G1, pTa. However, the cancer recurred in the urinary bladder 3 months after TUR-BT. Radical cystectomy was performed, and the disease was considered to have been cured, but metastasis developed in the skin, lung, liver, and bone several months after surgery. Chemotherapy was ineffective, and the patient died. On pathological examination at the time of radical cystectomy, the lesion was sarcomatoid carcinoma of the urinary bladder. Although the prognosis associated with this carcinoma is known to be poor, the possibility of underestimation on preoperative staging cannot be ruled out from the cause, in addition to the insufficiency of the current therapeutic strategy.

\section{Keywords}

\section{Sarcomatoid Carcinoma, Bladder Cancer}

\section{Introduction}

Bladder carcinomas are generally urothelial carcinomas. However, bladder carcinomas such as sarcomas associated with a poor prognosis are sometimes encountered. We report a 62-year-old female with a sarcomatoid carcinoma of the urinary bladder.

\section{Case Presentation}

The patient was a 62-year-old female with a past history of transurethral resection of a bladder tumor (TUR-Bt)

"Corresponding author. 
performed in March 2010. The tumor was papillary and had a diameter of $3 \mathrm{~cm}$, situated besides the left orifice. It was resected completely, and the surgical pathology at the time of TUR-Bt was urothelial carcinoma G2 > G1, pTa.

She visited our hospital for follow-up in July 2010. At her first visit, physical and blood examinations were normal, but microhematuria was noted. Ultrasonography, cystoscopy, and CT revealed a 3-cm tumor existing at the left orifice with hydronephrosis (Figure 1). We diagnosed her with invasive bladder cancer invading the left ureter, and performed radical cystectomy in August 2010. The pathological diagnosis was infiltrating urothelial carcinoma, sarcomatoid variant, G3, INF $\gamma$, pT3b, u-rt0, u-lt0, ur0, ew0, ly0, v0, n0 (0/9) (Figure 2).

The clinical course after cystectomy was rapid. One month after the operation, metastasis was noted in the skin on the top of the head, at 2 months it was noted in the liver and lung, and multiple bone metastases occurred at 3 months. CA19-9 was a useful tumor marker, and elevated up to 4659.6 U/mL. Systemic chemotherapy (GC: Gemcitabine + Cisplatin) was performed immediately. However, imaging examinations clearly revealed the progression of disease (PD) after a single course of chemotherapy, and so systemic chemotherapy was changed to another regimen (TJ: Paclitaxel + Carboplatin). After two courses of the chemotherapy, the disease progressed with a further elevation of CA19-9 to 99, $111.7 \mathrm{U} / \mathrm{mL}$. Subsequently, the general condition worsened and she died in February 2011.

\section{Discussion}

Some malignant tumors arising from the urinary bladder show sarcoma-like growth and are termed sarcomatoid carcinoma. Its incidence in all malignant bladder tumor cases is very low $(0.31 \%)$ [1]. Its characteristics are as follows: 1) Frequent onset age of 70 years or older, 2) rate of malignancy is high, 3) chemotherapy and radiotherapy are ineffective, and surgical resection is the first-line treatment, and 4) the prognosis is very poor [2]-[6]. The one-year survival rate has been reported to be less than 30\% [7]-[11].

Malignant tumors comprised of a mixture of vesical epithelial and sarcomatoid components were reported with various names, such as carcinosarcoma and malignant mixed tumors, in addition to sarcomatoid carcinoma [12] [13], suggesting that it is difficult to diagnose.

The spindle-cell component of sarcomatoid carcinoma was previously considered to be an epithelial tumor with epithelial component properties both immunohistologically and morphologically, whereas that of carcinosarcoma was considered a mesenchymal tumor with no epithelial component property, but no significant difference to aid in distinguishing between these was observed in a recent immunohistological investigation. In a study employing comparative genomic hybridization, epithelial and spindle-cell components of sarcomatoid carcinoma and carcinosarcoma showed a monoclonal origin [14]. Thompson et al. [15] also reported that epi-

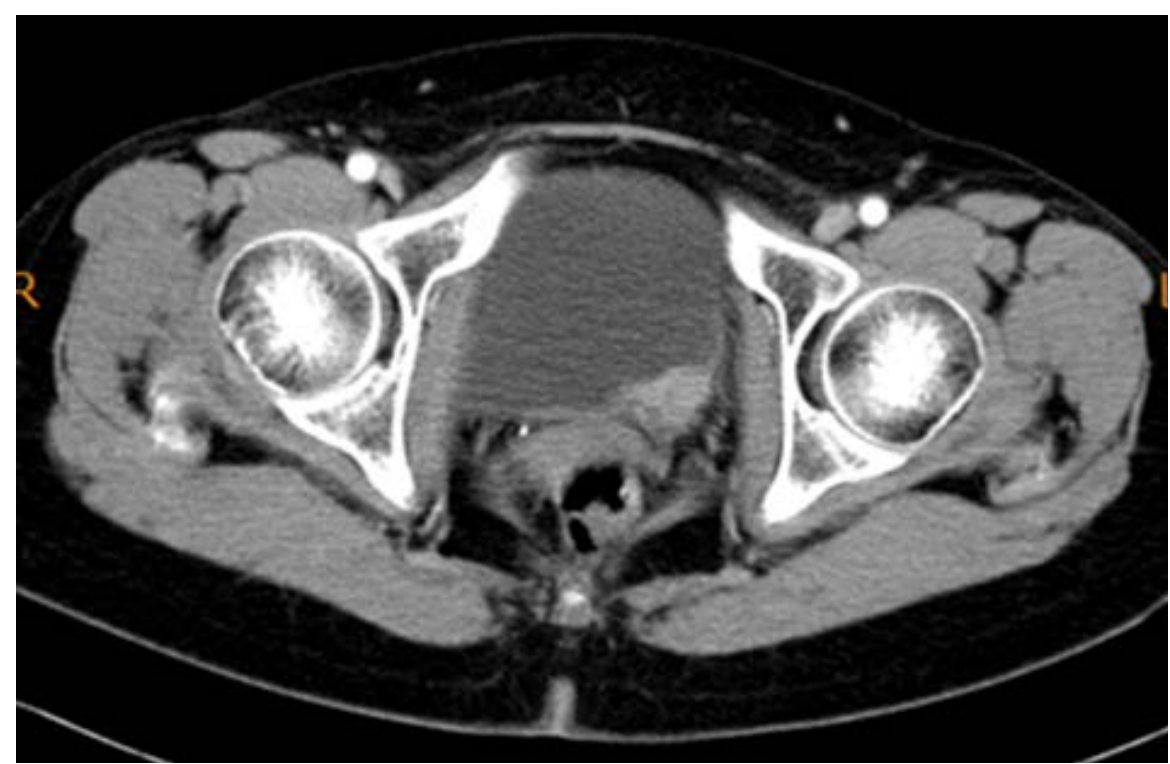

Figure 1. Bladder tumor is observed at the left orifice by CT. 
thelial and spindle-cell components were monoclonal on analysis of DNA clonality. They assumed that gene mutation occurred in an earlier phase of carcinogenesis in sarcomatoid carcinoma with residual spindle-cell and epithelial component properties than in carcinosarcoma. It was also reported that cancer and sarcomatoid components of sarcomatoid carcinoma of the urinary bladder were derived from common cells, i.e., monoclonal [16], and the sarcomatoid component of sarcomatoid carcinoma results from dedifferentiation, which occurs in association with the cell adhesion molecule-losing process [11]. Based on these reports, it is generally considered that, histopathologically, sarcomatoid carcinoma and carcinosarcoma are the same tumor type derived from epithelial components.

The total results of 42 Japanese cases combining those reported in 2007 by Kawamura et al. [17] and our patients are shown in Table 1. Radical or partial cystectomy was performed in 69.1\%, and TUR-Bt was performed in $30.9 \%$. The radical cystectomy group may have been treated so because the disease was definitely diagnosed pathologically, and cure was expected because of the absence of metastasis. The TUR-Bt group may have been incidentally diagnosed with sarcomatoid carcinoma pathologically. However, the mortality rate was $28.6 \%$ and the outcome was unclear in 21.4\%, i.e., 1 in 2 patients died, suggesting that 5 of 7 patients treated with radical cystectomy may die.

Our patient was also treated with an operation with curative intent but died soon after the surgery. Since sarcomatoid carcinoma of the urinary bladder grows rapidly, in addition to strict follow-up, the establishment of a new therapeutic strategy is necessary to deal with recurrence, for which the accumulation and investigation of cases are necessary. However, the possibility of underestimation of the preoperative stage cannot be ruled out.

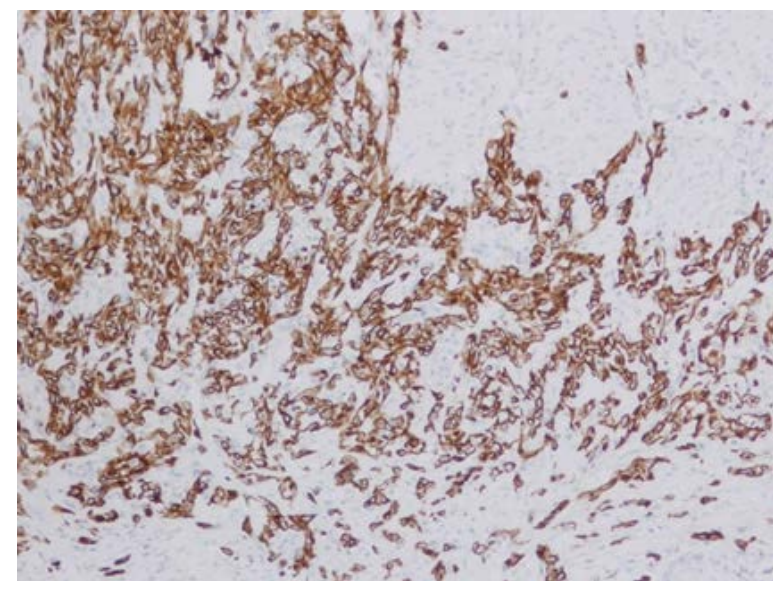

(a)

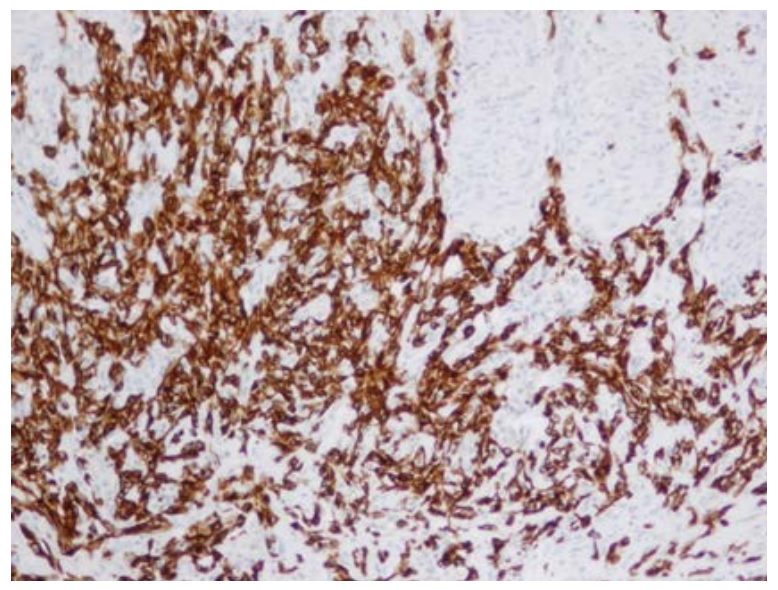

(b)

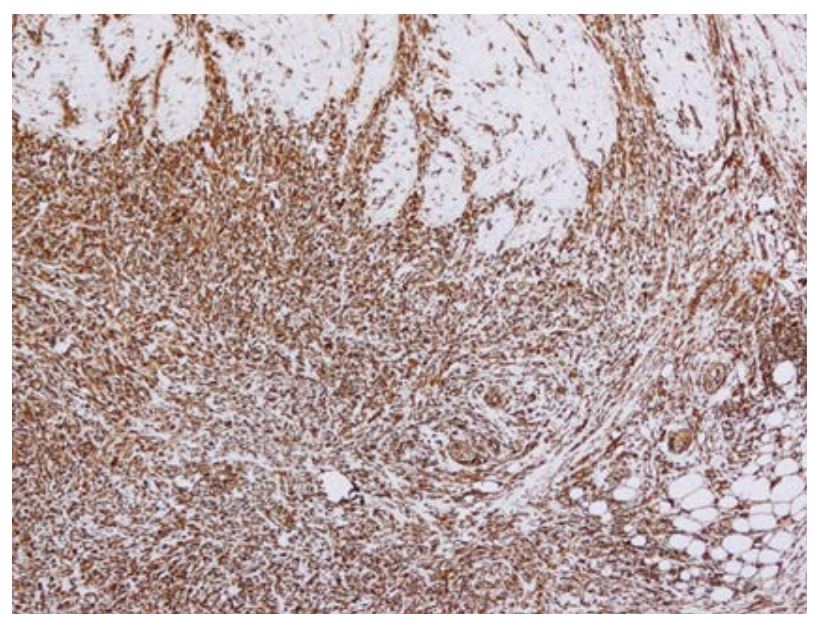

(c)

Figure 2. Microscopic photograohs. Sarcomatoid component was stained by AE1/AE3, CK7 and vimentin. (a) AE1/ AE3 × 100; (b) CK7 × 100; (c) vimentin $\times 40$. 
Table 1.42 cases of sarcomatoid carcinoma of the urinary bladder in Japan.

\begin{tabular}{|c|c|c|c|}
\hline & & No. of cases & $\%$ \\
\hline \multirow[t]{7}{*}{ Treatment } & Radical Cystectomy & 14 & 33.3 \\
\hline & Partial Cystectomy & 3 & 7.1 \\
\hline & Radical Cystectomy + Cx & 7 & 16.7 \\
\hline & Radical Cystectomy + Cx + Rad & 3 & 7.1 \\
\hline & Partial Cystectomy + Cx & 2 & 4.8 \\
\hline & TUR & 10 & 23.8 \\
\hline & $\mathrm{TUR}+\mathrm{Cx}+\mathrm{Rad}$ & 3 & 7.1 \\
\hline \multirow[t]{5}{*}{ T stage } & $\mathrm{T} 1$ & 4 & 9.5 \\
\hline & $\mathrm{T} 2$ & 11 & 26.2 \\
\hline & $\mathrm{T} 3$ & 14 & 33.3 \\
\hline & $\mathrm{T} 4$ & 2 & 4.8 \\
\hline & Unknown & 11 & 26.2 \\
\hline \multirow[t]{5}{*}{ Prognosis } & Alive without recurrence & 16 & 38.1 \\
\hline & Alive with recurrence & 3 & 7.1 \\
\hline & Dead & 12 & 28.6 \\
\hline & Recent cases & 2 & 4.8 \\
\hline & Unknown & 9 & 21.4 \\
\hline
\end{tabular}

Cx: Chemotherapy; Rad: Radiation.

Systemic staging using such as FDG-PET may also be necessary.

\section{References}

[1] Torenbeek, R., Blomjous, C.E., de Bruin, P.C., Newling, D.W. and Meijer, C.J. (1994) Sarcomatoid Carcinoma of the Urinary Bladder: Clinicopathologic Analysis of 18 Cases with Immunohistochemical and Electron Microscopic Findings. The American Journal of Surgical Pathology, 18, 241-249. http://dx.doi.org/10.1097/00000478-199403000-00003

[2] Miwa, K., Kamei, S., Nishino, Y., Takahashi, Y. and Degichi, T. (2000) Sarcomatoid Carcinoma of the Urinary Bladder. Acta Urological Japonica, 46, 193-196.

[3] Sumura, M., Yokogi, H. and Maruyama, R. (2002) Sarcomatoid Carcinoma of the Bladder. The Nishinihon Journal of Urology, 64, 472-476.

[4] Nagata, Y., Hashimoto, T. and Kawakami, T. (1995) Primary Bladder Carcinosarcoma: A Case Report and Review of 21 Cases Noted in Japanese Literature. Japanese Journal of Urological Surgery, 8, 223-225.

[5] Kaneko, H., Nakauchi, K., Takubo, K. and Ezaki, I. (1994) Sarcomatoid Carcinoma of the Urinary Bladder Associated with Other Malignancies: Three Case Reports. The Nishinihon Journal of Urology, 56, 1363-1367.

[6] Murao, T., Tanahashi, T. and Matsumura, Y. (1989) A Case of Vesical Carcinoma with a Sarcomatous Change-Immunohistochemical and Electron Microscopic Observations. Japanese Journal of Clinical Oncology, 35, 114-119.

[7] Moriyama, H., Kasaoka, Y., Fukushige, M., Asano, K., Nishizaka, T., Fukuhara, T. and Igawa, M. (1995) Sarcomatoid Carcinoma of the Bladder. The Nishinihon Journal of Urology, 57, 678-682.

[8] Young, R.H., Wick, M.R. and Mills, S.E. (1988) Sarcomatoid Carcinoma of the Urinary Bladder. A Clinicopathologic Analysis of 12 Cases and Review of the Literature. American Journal of Clinical Pathology, 90, 653-661.

[9] Ikegami, H., Iwasaki, H., Ohjimi, Y., Takeuchi, T., Ariyoshi, A. and Kikuchi, M. (2000) Sarcomatoid Carcinoma of the Urinary Bladder: A Clinicopathologic and Immunohistochemical Analysis of 14 Patienrs. Human Pathology, 31, 332-340. http://dx.doi.org/10.1016/S0046-8177(00)80247-6 
[10] Hasui, Y., Nishi, S., Kitada, S., Osada, Y. and Sumiyoshi, A. (1991) Comparative Immunohistochemistry of Malignant Fibrous Histiocytoma and Sarcomatoid Carcinoma of the Urinary Tract. Urological Research, 19, 69-72. http://dx.doi.org/10.1007/BF00294025

[11] Lopez-Beltran, A., Pacelli, A., Rothenberg, H.J., Wollan, P.C., Zincke, H., Blute, M.L. and Bostwick, D.G. (1998) Carcinosarcoma and Sarcomatoid Carcinoma of the Baladder: Clinicopathological Study of 41 Cases. Journal of Urology, 159, 1497-1503. http://dx.doi.org/10.1097/00005392-199805000-00023

[12] Masuda, H., Yamada, T., Nagahama, K., Nagamatsu, H. and Negishi, T. (1996) A Case of Carcinosarcoma of the Bladder. Japanese Journal of Urological Surgery, 9, 1083-1085.

[13] Young, R.H. (1987) Carcinosarcoma of the Urinary Bladder. Cancer, 59, 1333-1339. http://dx.doi.org/10.1002/1097-0142(19870401)59:7<1333::AID-CNCR2820590717>3.0.CO;2-N

[14] Torenbeek, R., Hermsen, M.A., Meijer, G.A., Baak, J.P. and Meijer, C.J. (1999) Analysis by Comparative Genomic Hybridization of Epithelial and Spindle Cell Components in Sarcomatoid Carcinoma and Carcinosarcoma: Histogenetic Aspects. Journal of Pathology, 189, 338-343. http://dx.doi.org/10.1002/(SICI)1096-9896(199911)189:3<338::AID-PATH429>3.0.CO;2-Q

[15] Thompson, L., Chang, B. and Barsky, S.H. (1996) Monoclonal Origins of Malignant Mixed Tumors (Carcinosarcoma). Evidence for a Divergent Histogenesis. The American Journal of Surgical Pathology, 20, 277-285. http://dx.doi.org/10.1097/00000478-199603000-00003

[16] Halachmi, S., DeMarzo, A.M., Chow, N.H., Halachmi, N., Smith, A.E., Linn, J.F., Nativ, O., Epstein, J.I., Schoenberg, M.P. and Sidransky, D. (2000) Genetic Anterations in Urinary Bladder Carcinosarcoma: Evidence of a Common Clonal Origin. European Urology, 37, 350-357. http://dx.doi.org/10.1159/000052369

[17] Kawamura, N., Kakuta, Y., Fukuhara, S., Imazu, T., Hara, T., Yamaguchi, S. and Adachi, S. (2007) Sarcomatoid Carcinoma of the Urinary Bladder. Acta Urological Japonica, 53, 713-716. 